\title{
Large renal carbuncle mimicking intra-abdominal neoplasm on Ga-67 scintigraphy: A case of fever of unknown origin
}

\author{
Yu-Hung Chen \\ Department of Nuclear Medicine, Buddhist Tzu Chi General Hospital, Hualien, Taiwan; \\ Corresponding Author: jedimasterchen@hotmail.com
}

Received 18 March 2013; revised 19 April 2013; accepted 7 May 2013

Copyright (C) 2013 Yu-Hung Chen. This is an open access article distributed under the Creative Commons Attribution License, which permits unrestricted use, distribution, and reproduction in any medium, provided the original work is properly cited.

\begin{abstract}
Fever of unknown origin is still a medical challenge. ${ }^{67} \mathrm{Ga}$ single photon emission computed tomography/computed tomography images are commonly used to evaluate the final diagnosis of fever of unknown origin. We presented a case of fever of unknown origin undergone ${ }^{67} \mathrm{Ga}$ scintigraphy and an intra-abdominal ${ }^{67} \mathrm{Ga}$ avid tumor was detected which suspected to be a neoplasm. Further contrast enhanced computed tomography revealed that the lesion was a large renal carbuncle. We concluded that the contrast-enhanced CT or single photon emission computed tomography/computed tomography with contrast enhancement can be performed to further improve diagnostic performance.
\end{abstract}

Keywords: Fever of Unknown Origin; Gallium Scintigraphy; Neoplasm; Renal Carbuncle

\section{INTRODUCTION}

Fever of unknown origin (FUO) remains to be a medical challenge despite nowadays technical improvements. It has evolved with time and now has covered wider diagnostic entities including HIV-associated FUO, neutropenic FUO and nosocomial FUO [1]. The diagnosis is made if three outpatient visits or 3 days in the hospital without elucidation of a cause or 1 week of intelligent and invasive ambulatory investigation [1,2]. Despite subsequent evaluations by many tools, up to $50 \%$ of cases with FUO reach no final diagnosis [2]. Literatures have indicated that various nuclear medicine images play an important role in investigating FUO [3], among these diagnostic images, ${ }^{67} \mathrm{Ga}$ scintigraphy is a valuable examination and is still used widely [4]. This is because that both inflammatory disorders and some neoplasms can be detected by ${ }^{67} \mathrm{Ga}$ scintigraphy and FUO may be caused by various malignancies. However, this may also tell us that we may not be able to differentiate inflammation from neoplasm on ${ }^{67} \mathrm{Ga}$ scintigraphy. We reported a case with FUO underwent ${ }^{67} \mathrm{Ga}$ scintigraphy with ${ }^{67} \mathrm{Ga}$ avid renal carbuncle mimicking an intra-abdominal neoplasm.

\section{CASE REPORT}

A 51-year-old man complained of fever for 3 weeks despite out-patient investigations. The cerebrospinal fluid analysis, chest films and abdominal ultrasonography showed negative results. A serum C-reactive protein was $13.86 \mathrm{mg} / \mathrm{dL}$. ${ }^{67} \mathrm{Ga}$ whole body scintigraphy was arranged and the images were taken 65 hours after $6.1 \mathrm{mCi}$ (225.7 MBq) intravenous ${ }^{67} \mathrm{Ga}$ citrate. The planar image revealed dense ${ }^{67} \mathrm{Ga}$ accumulation in left upper abdomen (Figure 1(a)) and the single photon emission computed tomography/computed tomography (SPECT/ CT) revealed that the radiotracers were located at a tu- mor (Figures 1(b) and (c)). It was unable to differenti- ate whether it was renal origin or not. Subsequent ab- dominal contrast-enhanced computed tomography (CT) revealed that the ${ }^{67} \mathrm{Ga}$ avid tumor was a renal cyst (Figures 2(a) and (b)) and a renal calculus was also noticed in the cyst. Previous abdominal CT also demonstrated the renal calculus, however, the cyst was smaller. A midstream urine culture yielded Escherichia coli of 7000 colony forming units per $\mathrm{mL}$. The patient received daily intravenous ceftriaxone $2000 \mathrm{mg}$ and he defeverized after the antibiotic treatment.

\section{DISCUSSION}

${ }^{67} \mathrm{Ga}$ scintigraphy is a common clinical strategy for pa- 


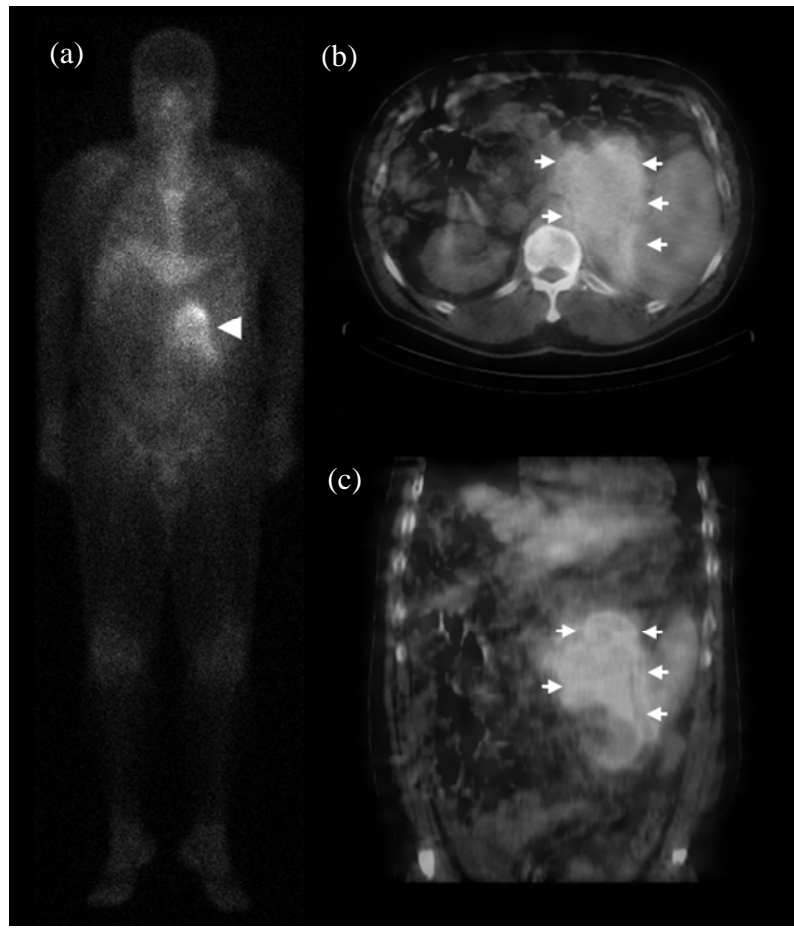

Figure 1. ${ }^{67} \mathrm{Ga}$ whole body scintigraphy: Planar image (a) revealed dense heterogeneous ${ }^{67} \mathrm{Ga}$ accumulation in left upper abdomen (Arrowhead). The trans-axial (b) and coronal (c) SPECT/CT images demonstrated that the ${ }^{67} \mathrm{Ga}$ avid lesion was located at a tumor above or at left kidney (Arrow).

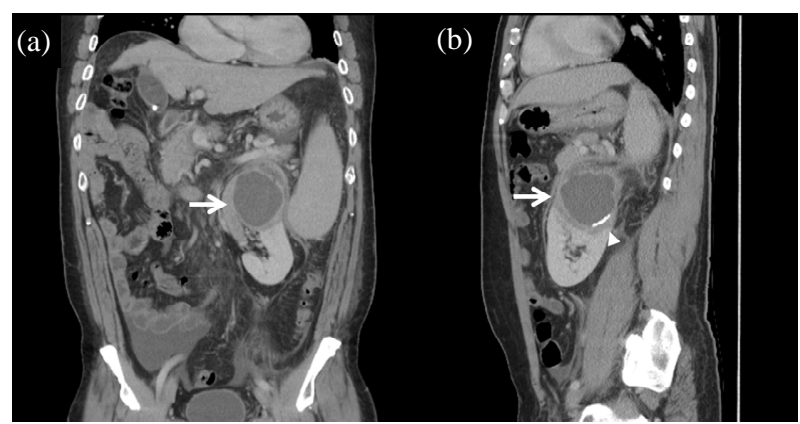

Figure 2. The contrast enhanced computed tomography in coronal section (a) and sagittal section (b) showed a large renal cyst measured to be $7 \times 6 \mathrm{~cm}$ (Arrow) at upper pole of left kidney, there were many small cystic lesions in the capsule. There was a nephrolithiasis (Arrowhead) in the sagittal section.

tients with FUO. ${ }^{67} \mathrm{Ga}$ behaves like iron and can bind to lactoferrin and siderophore, molecules present at site of inflammation and many neoplasms. According to the literature, ${ }^{67} \mathrm{Ga}$ scintigraphy can be helpful in diagnostic evaluation in $29 \%$ of cases with FUO [4]. Among the patients reach final diagnosis from ${ }^{67} \mathrm{Ga}$ scintigraphy, mostly (54\%) are infectious, followed by neoplasm (19\%) and inflammatory or rheumatologic (16\%) [5]. Modern use of integrated SPECT/CT can significantly improve
$26 \%$ of scan interpretation compared to planar image or SPECT image along [6]. Evidence also suggested that the application of SPECT/CT on ${ }^{67} \mathrm{Ga}$ scintigraphy can further improve diagnosis, localization and definition of extent of disease [7]. In our patient, SPECT/CT localized the lesion successfully; however, the images provided insufficient information to determine the nature of tumor. Further contrast-enhanced CT gave more detailed tissue vascularity information and the final diagnosis was made. Although the integration of CT into SPECT provides anatomical information to functional ${ }^{67} \mathrm{Ga}$ images, noncontrast enhanced CT lakes the information of vascularrity, which is important in differentiating different type of tissues. Thus, with addition of contrast enhanced CT or even performing SPECT/CT with contrast enhancement may further improve the diagnostic performance of FUO.

\section{REFERENCES}

[1] Knockaert, D.C., Vanderschueren, S. and Blockmans, D. (2003) Fever of unknown origin in adults: 40 years on. Journal of Internal Medicine, 253, 263-275. doi:10.1046/j.1365-2796.2003.01120.x

[2] Bleeker-Rovers, C.P., van der Meer, J.W. and Oyen, W.J. (2009) Fever of unknown origin. Seminars in Nuclear Medicine, 39, 81-87.

doi:10.1053/j.semnuclmed.2008.10.002

[3] Gaeta, G.B., Fusco, F.M. and Nardiello, S. (2006) Fever of unknown origin: A systematic review of the literature for 1995-2004. Nuclear Medicine Communications, 27, 205-211. doi:10.1097/00006231-200603000-00002

[4] Knockaert, D.C., Mortelmans, L.A., De Roo, M.C. and Bobbaers, H.J. (1994) Clinical value of gallium-67 scintigraphy in evaluation of fever of unknown origin. Clinical Infectious Disease, 18, 601-605. doi:10.1093/clinids/18.4.601

[5] Habib, G.S., Masri, R. and Ben-Haim, S. (2004) The utility of gallium scintigraphy in the evaluation of fever of unknown origin. Israel Medical Association Journal, 6, 463-466.

[6] Roach, P.J., Schembri, G.P., Ho Shon, I.A., Bailey, E.A. and Bailey, D.L. (2006) SPECT/CT imaging using a spiral CT scanner for anatomical localization: Impact on diagnostic accuracy and reporter confidence in clinical practice. Nuclear Medicine Communications, 27, 977-987. doi:10.1097/01.mnm.0000243372.26507.e7

[7] Bar-Shalom, R., Yefremov, N., Guralnik, L., Keidar, Z., Engel, A., Nitecki, S., et al. (2006) SPECT/CT using 67Ga and 111In-labeled leukocyte scintigraphy for diagnosis of infection. Journal of Nuclear Medicine, 47, 587594. 